\title{
Per ardua ad astra
}

\section{Washington}

DESPITE a troublesome start, the orbiting astronomy package Astro-1, a set of telescopes tucked into the cargo bay of space shuttle Columbia, was pronounced a great success halfway through its ten-day mission.

The threat of a premature end to the mission, after the second of two instrument display units in the shuttle cabin failed, was averted when control of the three ultraviolet and one X-ray telescopes was transferred to the Marshall Space Flight Center (MSFC) in Huntsville, Alabama. A makeshift arrangement, with astronauts steering the telescopes manually and scientists at Marshall operating them, was working smoothly in less than a day. Last weekend, there was some hope that the lost day could be recovered by the extension of the mission to an eleventh day but over the weekend a waste liquid tube became clogged, and it seemed as if the shuttle would have to return to Earth early. By last Monday the plumbing was fixed, but by then the threat of bad weather in California and Florida forced a decision to land early anyway.

The Astro-1 observatory was due to have been launched in May, but was delayed by the plague of hydrogen leaks that afflicted the shuttle fleet throughout the summer. Then, after the successful launch of Columbia on 25 November, a string of problems hinted that Astro-1's ill-fortune might continue.

One of two units displaying the status of the astronomy package failed altogether after nine hours, apparently due to overheating. A partial computer failure in the Wisconsin Ultraviolet Photo-Polarimeter Experiment (WUPPE) set back attempts jointly to align all three ultraviolet telescopes, and a later computer crash disabled an optical tracking device needed to lock on to guide stars and maintain the telescopes' pointing accuracy.

But all these setbacks proved temporary or manageable. By the third day of the mission, all four telescopes, including the separately steerable Broad-Band Xray Telescope (BBXRT), were working satisfactorily and, with manual help from a mission specialist operating an on-board joystick, were being pointed successfully and held on target.

But after a full day of observation, the second of the two instrument display units failed, apparently also from overheating. This left the astronauts able to steer the telescopes, but unable to perform the necessary switching of filters and management of exposure time.

Throughout last Thursday, mission scientists worked to re-route control of telescope operations to MSFC, and were able to try out a first version of the new operating regime barely 12 hours after the failure. By Thursday evening, Astro-1 was up and running again. At the same time, the observing schedule was reorganized: each 12-hour block of observing time was planned just 12 hours in advance.

With control of the observatory now in the hands of principal investigators at MSFS rather than the mission specialists on Columbia, some astronomers say that the new mode of operation is preferable to that originally planned.

Bill O'Connell, of the Ultraviolet Imaging Telescope (UIT) team, says that, in general, fewer objects are now being observed for longer, which in fact meant more science for UIT, a camera whose film will not be developed until after Columbia returns. In the original plan, there were many short observations which enabled WUPPE and HUT to take quick looks at a variety of interesting objects, but which were too short to allow UIT to obtain useful images.

According to Art Davidsen, of the HUT team, all the highest priority objects for their instrument would be successfully rescheduled. Halfway through the mis-

sion, HUT had already obtained "lots of good stuff" on quasars and galaxies. One of the most exciting possibilities for Astro1 's ultraviolet observations, which reach to short wavelengths never before observed, would be to gather evidence for emission from extremely hot gas very close to the giant black holes thought to power quasars and active galaxies.

Steve Maran, of the UIT team, called Astro-1 an "extraordinary success", and said that operations were becoming more efficient with every day. The fact that the mission had recovered so rapidly and completely from a variety of difficulties argued in favour of this kind of science effort on the shuttle, in which observers operating their own instruments could adapt quickly to changing circumstances.

Whether there will indeed be more has yet to be decided by the National Aeronautics and Space Administration (NASA). A new shuttle manifest, covering the next three years, was released last week, with no spot for a reflight of Astro1. But Davidsen is hopeful that astronomers will push hard to make it fly again, and he hopes that even NASA "will budge when they see the results."

David Lindley

\section{Telescope high and dry in the desert}

\section{Munich}

ONE of the clearest, driest places in the world was chosen last week by the European Southern Observatory (ESO) as the site for its Very Large Telescope (VLT). Cerro Paranal, a 2,664-metre-high mountain in the Atacama Desert in Chile, was 品

\section{IMAGE UNAVAILABLE FOR COPYRIGHT REASONS}

\section{The best site for sight.}

selected for the excellent image quality and good weather as well as the absence of light pollution.

A six-year meteorological survey showed that Paranal is one of the best sites in the world for optical astronomy, approaching the standards of Mauna Kea, Hawaii, generally considered the best year-round site for optical and infrared astronomy. Several US and international telescopes are based there.

Although Mauna Kea has the advantage of being at a higher altitude, so that less atmospheric water vapour interferes with spectroscopic measurements in the infrared, Paranal has more clear nights and a more stable atmosphere. In terms of 'seeing', meaning the sharpness of stellar objects in both optical and infrared astronomy, Paranal will be better (at a median of 0.66 arcseconds) than the main ESO facility in Chile at La Silla (median of 0.76 arcseconds), which is well known for its excellent observing conditions. Similar measurements are not yet available for seeing at Mauna Kea.

The only surprise about the decision was the placement of the telescope so far from La Silla, $600 \mathrm{~km}$ to the south. ESO astronomer Massimo Tarenghi was confident that no logistical difficulties would arise from the separation.

VLT consists of four 8.2-metre telescopes which can work separately or together, in which case they make VLT with its effective 16-metre diameter the largest optical telescope in the world. The four telescopes will begin to be installed in 1995 and the entire array should be in place by late 1998 .

There will be a satellite link between Paranal and ESO headquarters in Garching, Germany, allowing astronomers to control VLT remotely. The high-speed link of up to 8 megabaud will allow even better remote control than is currently possible between Garching and La Silla.

Steven Dickman 